\title{
Article
}

\section{Effects of protein-pheromone complexation on correlated chemical shift modulations}

\author{
Chiara Perazzolo ${ }^{\mathrm{a}}$, Julien Wist ${ }^{\mathrm{a}}$, Karine Loth $^{\mathrm{c}}$, Luisa Poggi ${ }^{\mathrm{c}}$, Steve Homans ${ }^{\mathrm{b}}$ \& Geoffrey \\ Bodenhausen $^{\mathrm{a}, \mathrm{c}, *}$ \\ ${ }^{\mathrm{a}}$ Institut des Sciences et Ingénierie Chimiques, Ecole Polytechnique Fédérale de Lausanne, BCH, 1015 \\ Lausanne, Switzerland; ' ${ }^{\mathrm{b}}$ School of Biochemistry and Microbiology, University of Leeds, Woodhouse Lane, \\ LS2 9JT, Leeds, England; 'Département de chimie, associé au CNRS, Ecole Normale Supérieure, 24 rue \\ Lhomond, 75231 Paris cedex 05, France
}

Received 1 August 2005; Accepted 12 September 2005

Key words: chemical shift anisotropy, chemical shift modulations, cross-correlation rates, major urinary protein, slow protein backbone motions, slow protein dynamics

\begin{abstract}
Major urinary protein (MUP) is a pheromone-carrying protein of the lipocalin family. Previous studies by isothermal titration calorimetry (ITC) show that the affinity of MUP for the pheromone 2-methoxy-3isobutylpyrazine (IBMP) is mainly driven by enthalpy, with a small unfavourable entropic contribution. Entropic terms can be attributed in part to changes in internal motions of the protein upon binding. Slow internal motions can lead to correlated or anti-correlated modulations of the isotropic chemical shifts of carbonyl $\mathrm{C}^{\prime}$ and amide $\mathrm{N}$ nuclei. Correlated chemical shift modulations (CSM/CSM) in MUP have been determined by measuring differences of the transverse relaxation rates of zero- and double-quantum coherences $Z \mathrm{QQC}\left\{\mathrm{C}^{\prime} \mathrm{N}\right\}$ and $\mathrm{DQC}\left\{\mathrm{C}^{\prime} \mathrm{N}\right\}$, and by accounting for the effects of correlated fluctuations of dipole-dipole couplings (DD/DD) and chemical shift anisotropies (CSA/CSA). The latter can be predicted from tensor parameters of $\mathrm{C}^{\prime}$ and $\mathrm{N}$ nuclei that have been determined in earlier work. The effects of complexation on slow time-scale protein dynamics can be determined by comparing the temperature dependence of the relaxation rates of APO-MUP (i.e., without ligand) and HOLO-MUP (i.e., with IBMP as a ligand).
\end{abstract}

\section{Introduction}

Many macromolecular interactions occur with concomitant changes in enthalpy and entropy, reflecting the fundamental tendency of a system to seek its lowest Gibbs energy. Inspection of highresolution structures of protein complexes often reveals a large number of favourable interactions, like

*To whom correspondence should be addressed. E-mail: Geoffrey.Bodenhausen@ens.fr hydrogen bonds and closely-packed hydrophobic residues, which suggest that enthalpic contributions to binding must be significant. Yet intermolecular affinity can also be determined, at least in part, by entropic effects associated with changes in internal mobility, and recent work has underscored the importance of protein flexibility in governing affinity and specificity (Kay et al., 1998; Zídek et al., 1999; Lee et al., 2000; Krízová et al., 2004).

Mouse major urinary protein (MUP) is an abundant pheromone-binding protein found in 
male mouse urine, where subtle recognition of a series of related compounds is essential to biological function. Stone, Sklenar and their co-workers (Zídek et al., 1999; Krízová et al., 2004) have shown that the backbone flexibility undergoes a slight increase upon binding of MUP with a hydrophobic pheromone, 2-sec-butyl-4,5-dihydrothiazole. They monitored changes in flexibility at high frequencies in terms of backbone order parameters $S^{2}$, obtained by 'model-free' analysis (Lipari and Szabo, 1982a, b). These order parameters slightly decrease upon binding, so that the backbone conformational entropy appears to increase upon binding. Homans and co-workers (Bingham et al., 2004) conducted a thorough investigation of the thermodynamic aspects of the binding affinity of MUP to various ligands by isothermal titration calorimetry (ITC). The overall entropy $\Delta S$ was found to decrease upon binding, so that binding must be driven by enthalpy, but differences in binding affinity for different ligands may be explained in part by entropic effects. Homans and coworkers (Bingham et al., 2004) also applied the 'model-free' analysis (Lipari and Szabo, 1982a, b) to both APO-MUP and HOLOMUP, i.e., the complex with 2-methoxy-3-isobutylpyrazine (IBMP), and found that the overall backbone conformational entropy slightly decreases upon binding, in contrast to the findings of Stone, Sklenar and their co-workers (Zídek et al., 1999; Krízová et al., 2004). These contradictory observations call for a more detailed analysis of the changes in internal mobility upon complexation. Indeed, fast internal motions on nsps time scales, which can be picked up with backbone order parameters $S^{2}$, only represent a subset of all dynamical processes in a protein. Slow motions on $\mu$ s to ms time scales can lead to fluctuations of the isotropic chemical shifts, leading to so-called $R_{\text {ex }}$ contributions to the linewidths. Frueh et al. (2001) detected slow motions that simultaneously affect the shifts of both $\mathrm{C}^{\alpha}$ and $\mathrm{C}^{\beta}$ nuclei in the side-chains of proteins. The difference between the auto-relaxation rates of zeroquantum coherences $\mathrm{ZQC}\left(\mathrm{C}_{+}^{\alpha} \mathrm{C}_{-}^{\beta}\right)$ and doublequantum coherences $\mathrm{DQC}\left(\mathrm{C}_{+}^{\alpha} \bar{C}_{+}^{\beta}\right)$ is due to contributions from cross-correlated isotropic chemical shift modulations (CSM/CSM), crosscorrelated fluctuations of dipolar interactions (DD/DD) and cross-correlated anisotropic chemical shift interactions (CSA/CSA). More recently, Dittmer and Bodenhausen (2004) measured the relaxation rates of $\mathrm{ZQC}\left(\mathrm{N}_{+} \mathrm{H}_{-}^{\mathrm{N}}\right)$ and $\operatorname{DQC}\left(\mathrm{N}_{+} \mathrm{H}_{+}^{\mathrm{N}}\right)$ and Wist et al. (2005) those of $\mathrm{ZQC}\left(\mathrm{C}_{+}^{\prime} \mathrm{N}_{-}\right)$and DQC $\left(\mathrm{C}_{+}^{\prime} \mathrm{N}_{+}\right)$. The latter method appears most appropriate to reveal the effects on internal motions of binding of pheromones to MUP.

\section{Materials and methods}

Both APO-MUP and HOLO-MUP samples (Bingham et al., 2004) were dissolved in $10 \%$ $\mathrm{D}_{2} \mathrm{O} / 90 \% \mathrm{H}_{2} \mathrm{O}$, and the $\mathrm{pH}$ was adjusted to 7.4 with phosphate saline buffer. Sequential assignments were obtained by 3D HNCO/HNCA (Kay et al., 1990) and $\mathrm{CBCA}(\mathrm{CO}) \mathrm{NH}$ methods (Grzesiek and Bax, 1992; Muhandiram and Kay, 1994). Transverse ${ }^{15} \mathrm{~N}$ relaxation rates were measured with de- and re-focusing intervals $\tau=1 \mathrm{~ms}$. The details of the DQC and ZQC methods are described elsewhere (Pellecchia et al., 1999; Wist et al., 2004, 2005). The data were acquired at $T=308 \mathrm{~K}$ using Shigemi tubes with two Bruker DMX-600 spectrometers in Paris and Lausanne, both equipped with triple resonance TBI probes with triple axis gradients. A cryoprobe was used for the temperaturedependent study. For the DQC and ZQC methods, each matrix consisted of 64 and 512 points in the $t_{1}$ and $t_{2}$ dimensions. The spectral widths were 1824 and $8389 \mathrm{~Hz}$ in the $\omega_{1}$ and $\omega_{2}$ dimensions. For each $t_{1}$ increment 64 scans were accumulated with a relaxation delay of $1.2 \mathrm{~s}$. The relaxation intervals $T$ were $1,6,14,24$, and $36 \mathrm{~ms}$. The experimental time required for each 2D experiment was $2.5 \mathrm{~h}$. Data processing has been performed using the GNU Package NMRPipe/NMRDraw/NlinLS (Delaglio et al., 1995). Backbone assignment was carried out using the Sparky 3 software package (T. D. Goddard and D. G. Kneller, University of California, San Francisco, USA). Relaxation rates were obtained by least-squared fitting of the decays to exponential functions (Matlab, 1992, Matlab Reference Guide, Natick, Massachusetts). Errors of the decay rates were estimated by Monte-Carlo analysis using 500 synthetic data points. Errors in the rates were then obtained by error propagation. A table of rates is available in the Supplementary Material. 


\section{Theory}

To determine the auto-relaxation rates of $\mathrm{ZQC}\left(\mathrm{C}_{+}^{\prime} \mathrm{N}_{-}\right)$and $\operatorname{DQC}\left(\mathrm{C}_{+}^{\prime} \mathrm{N}_{+}\right)$, we used the pulse sequences (Wist et al., 2005) of Figure S1 (see Supplementary Material) that are closely related to experiments proposed by Zuiderweg and coworkers (Pellecchia et al., 1999), although in the present case they are used for monitoring internal dynamics in addition to CSA effects. For each pair of neighbouring residues $(i-1, i)$, one can determine the 'raw' difference of the experimental decay rates of $\mathrm{DQC}\left(\mathrm{C}_{+}^{\prime} \mathrm{N}_{+}\right)$and $\mathrm{ZQC}\left(\mathrm{C}_{+}^{\prime} \mathrm{N}_{-}\right)$:

$$
R_{i}^{\text {exp }}=1 / 2\left[R_{i}(\mathrm{DQC})-R_{i}(\mathrm{ZQC})\right]
$$

Relaxation theory shows that three cross-correlated fluctuations contribute to this rate (Frueh et al., 2001; Dittmer and Bodenhausen, 2004):

$$
R_{i}^{\text {theor }}=R_{i}^{\mathrm{CSM} / \mathrm{CSM}}+R_{i}^{\mathrm{CSA} / \mathrm{CSA}}+R_{i}^{\mathrm{DD} / \mathrm{DD}}
$$

The first two terms are proportional to $\mathrm{B}_{0}^{2}$, while the third one is essentially field independent. The CSA/CSA and DD/DD cross-correlation terms (the latter comprising sums over all pairs of neighbouring nuclei) are roughly proportional to $\tau_{\mathrm{c}}$ (which is on the order of $10 \mathrm{~ns}$ ), while the CSM/ CSM terms depend only on slow internal motions with a time-scale $\tau_{\text {int }}$ (typically in a range between $\mu$ s and $\mathrm{ms}$ ). These rates arise from slow modulations of the isotropic chemical shifts of $\mathrm{C}^{\prime}$ and $\mathrm{N}$ that are due to structural fluctuations, notably to variations of the dihedral angles $\phi$ and $\psi$ in the protein backbone, as shown by ab initio calculations (Arnold and Oldfield, 2000). Only when $\tau_{\text {int }} \gg \tau_{\mathrm{c}}$ do the resulting modulations of the isotropic shifts affect the relaxation of the coherences $\mathrm{ZQC}\left(\mathrm{C}_{+}^{\prime} \mathrm{N}_{-}\right)$and $\mathrm{DQC}\left(\mathrm{C}_{+}^{\prime} \mathrm{N}_{+}\right)$(Frueh et al., 2001).

The ${ }^{15} \mathrm{~N} /{ }^{1} \mathrm{H}$ HSQC spectra of the APO and HOLO forms (see Supplementary Material) reveal surprisingly large shifts for many residues. The published X-ray diffraction studies of the APO and HOLO forms (PDB entry codes Berman et al., 2000: 1QY0 and 1QY1) seem to indicate that the structures are very similar. In fact however, the APO form used for crystallography was not truly empty, since the binding site was occupied by a cryoprotectant (Bingham et al., 2004). Accurate measurements of ${ }^{15} \mathrm{~N} R_{1}$ and $R_{2}$ relaxation rates in two laboratories (Paris and Lausanne) show that the overall tumbling correlation time is $\tau_{\mathrm{c}}=8.9 \mathrm{~ns}$ for HOLO-MUP and $\tau_{\mathrm{c}}=7.1 \mathrm{~ns}$ for APO-MUP at $308 \mathrm{~K}$. These measurements stand in contrast to earlier findings by Sklenar and co-workers (Krízová et al., 2004) (who considered a different pheromone) and by Bingham et al. (2004) (who used lower concentrations and hence had larger errors). A two-fold dilution of the HOLO-MUP sample does not lead to any difference in $\tau_{\mathrm{c}}$, so that a propensity for partial dimerization can be excluded (Poggi, L., unpublished data). The reasons for the discrepancy between the present work and that from other laboratories are under investigation. Meantime, however, small differences in structures possibly implied by the rotational correlation times do not have an adverse bearing on the flow of our arguments, since accurate knowledge of the structures is not essential (vide infra).

The dipolar contributions to the decay rates of the $\mathrm{DQC}\left(\mathrm{C}_{+}^{\prime} \mathrm{N}_{+}\right)$and $\mathrm{ZQC}\left(\mathrm{C}_{+}^{\prime} \mathrm{N}_{-}\right)$coherences are due to a combination of auto- and cross-correlated effects (Frueh, 2002). The average autocorrelated $\mathrm{C}^{\prime}-\mathrm{N} / \mathrm{C}^{\prime}-\mathrm{N}$ dipole-dipole rates can be estimated to be $\left\langle R^{\mathrm{DD} / \mathrm{DD}}\right\rangle=0.027 \mathrm{~s}^{-1}$ in HOLOMUP $\left(\tau_{\mathrm{c}}=8.9 \mathrm{~ns}\right.$ at $\left.308 \mathrm{~K}\right)$ and $\left\langle R^{\mathrm{DD} / \mathrm{DD}}\right\rangle$ $=0.033 \mathrm{~s}^{-1}$ in APO-MUP $\left(\tau_{\mathrm{c}}=7.1 \mathrm{~ns}\right.$ at $\left.308 \mathrm{~K}\right)$, making the worst-case assumption that the backbone is rigid (all local order parameters set to $S_{\mathrm{i}}^{2}=1$ ). These auto-correlated DD contributions can thus be safely neglected. On the other hand, several cross-correlated dipolar mechanisms that involve external nuclei may contribute to $R_{i}^{\mathrm{DD} / \mathrm{DD}}$, i.e., $\mathrm{C}^{\prime}-\mathrm{X} / \mathrm{N}-\mathrm{X}$ or $\mathrm{C}^{\prime}-\mathrm{X} / \mathrm{N}-\mathrm{Y}$, where $\mathrm{X}$ and $\mathrm{Y}$ can stand for a proton $\left(\mathrm{H}^{\mathrm{N}}, \mathrm{H}^{\alpha}, \mathrm{H}^{\beta}\right.$, etc.) or a carbon13 nucleus (e.g., $\mathrm{C}^{\alpha}$ ). Using bond distances and angles extracted from the X-ray structures of the HOLO form, we estimated the cross-correlated contributions $R^{\mathrm{DD} / \mathrm{DD}}$ at $308 \mathrm{~K}$ (represented by red bars in the histogram of Figure S3 and Table S3, Supplementary Material). For most residues, the main contributions to this rate are due to the amide proton $\left(\mathrm{X}=\mathrm{H}^{\mathrm{N}}\right)$, so that the estimated rates $R^{\mathrm{DD} / \mathrm{DD}}$ do not critically depend on the precise knowledge of the structure (Bytchenkoff et al., 2005).

The CSA/CSA contributions in Equation (2) are also affected by complexation, since both the 
correlation time $\tau_{\mathrm{c}}$ and the CSA tensors of many $\mathrm{C}^{\prime}$ and $\mathrm{N}$ nuclei are significantly modified upon complexation. The HSQC spectrum in Figure S2 (Supplementary Material) shows that the isotropic shifts of many $\mathrm{N}$ and $\mathrm{H}^{\mathrm{N}}$ nuclei are affected. Similar changes are also observed for the isotropic $\mathrm{C}^{\prime}$ shifts (data not shown). A recent study of 14 complementary cross- and auto-correlation rates in Ubiquitin (Loth et al., 2005) indicates that some of the principal components of CSA tensors of $\mathrm{C}^{\prime}$, $\mathrm{N}$ and $\mathrm{H}^{\mathrm{N}}$ are nearly invariant from one aminoacid to another, while other components are proportional to the isotropic shifts. The site-specific CSA tensors have been determined in Ubiquitin using different motional models: (a) isotropic wobbling-in-a-cone; (b) three-dimensional Gaussian Axial Fluctuations (3D-GAF) with a common correlation time around all three orthogonal axes; (c) a model which allows the amide protons to move out of the peptide plane with an internal correlation time $\tau_{\text {int }}=40 \mathrm{ps}$; (d) the same model with $\tau_{\text {int }}=400 \mathrm{ps}$; and (e) the same as (d), but where the nitrogen CSA tensors are assumed to follow the motions of the amide protons. These five models lead to five slightly different estimates of the site-specific CSA tensors of the $\mathrm{C}^{\prime}$ and $\mathrm{N}$ nuclei. In accordance with Loth et al. (2005) we prefer the 3D-GAF model ('model b') to extract the CSA tensors of Ubiquitin. For the CSA tensors of $\mathrm{C}^{\prime}$ nuclei, the $\sigma_{y y}$ components are roughly proportional to the isotropic shifts $\left(\sigma_{y y} \approx 3 \sigma_{\text {iso }}-334.9 \mathrm{ppm}\right)$, while the other two components are almost invariant $\left(\sigma_{x x} \approx 251.2 \mathrm{ppm}\right.$; $\sigma_{z z} \approx 83.6 \mathrm{ppm}$ ). Likewise, for the CSA tensors of $\mathrm{N}$ nuclei, both $\sigma_{x x}$ and $\sigma_{y y}$ components are roughly proportional to the isotropic shifts $\left(\sigma_{x x} \approx \sigma_{\text {iso }}+105.5 \mathrm{ppm} ; \sigma_{y y} \approx 2 \sigma_{\text {iso }}-163.2 \mathrm{ppm}\right)$, while the third component is almost invariant $\left(\sigma_{z z} \approx 57.7 \mathrm{ppm}\right)$. These empirical rules, the details of which are given by Loth et al. (2005) could in principle be verified for MUP, but the consistency of our observations in Ubiquitin leads us to assume that the same relations can be used for APOand HOLO-MUP. The $R_{i}^{\mathrm{CSA} / \mathrm{CSA}}$ rates can thus be estimated for APO- and HOLO-MUP from the knowledge of the isotropic shifts and the correlation times, as represented by blue bars in the histogram of Figure S3 and Table S2 (Supplementary Material). Note that the knowledge of the structure is immaterial. We can now subtract the estimated rates $R_{i}^{\mathrm{CSA} / \mathrm{CSA}}$ and $R_{i}^{\mathrm{DD} / \mathrm{DD}}$ from the 'raw' experimental relaxation rates of Equation (1) to obtain the CSM/CSM contributions shown in Figure $2 b$ :

$$
R_{i}^{\mathrm{CSM} / \mathrm{CSM}}=R_{i}^{\exp }-R_{i}^{\mathrm{CSA} / \mathrm{CSA}}-R_{i}^{\mathrm{DD} / \mathrm{DD}}
$$

Note that the CSM/CSM cross-correlation rate on the left-hand side is a hybrid quantity, since the first term on the right-hand side represents 'raw' experimental rates, while the last two rates are estimated from (imperfect) knowledge of isotropic shifts, CSA tensors, and rotational correlation times, bearing in mind that uncertainties about the structure have little effect. Ideally, the CSM/CSM cross-correlation rate thus obtained should not be affected by any CSA and DD contributions, and be due only to slow fluctuations of the isotropic chemical shifts (see Equations $(88,89)$ in the work of Frueh, 2002). If the internal motions can be modelled (as usually assumed) in terms of instantaneous jumps between two different conformations $\mathrm{A}$ and $\mathrm{B}$ with populations $p_{\mathrm{A}}$ and $p_{\mathrm{B}}$, and if these jumps are fast enough so that one cannot distinguish two distinct resonances for the two conformations (traditionally called the 'fast exchange limit' of chemical exchange processes, although the exchange between conformations is of course much slower than the Larmor frequency), the rate can be described by (Frueh, 2002):

$$
R_{i}^{\mathrm{CSM} / \mathrm{CSM}}=2 p_{\mathrm{A}} p_{\mathrm{B}} \tau_{\mathrm{ex}} \Delta \omega_{\mathrm{AB}}^{\mathrm{C}^{\prime}} \Delta \omega_{\mathrm{AB}}^{\mathrm{N}}
$$

where $\tau_{\mathrm{ex}}=1 /\left(k_{\mathrm{AB}}+k_{\mathrm{BA}}\right)$ is inversely proportional to the sum of the forward and backward exchange rates between the conformations $\mathrm{A}$ and $\mathrm{B}$ in the APO form, and $\Delta \omega_{\mathrm{AB}}^{\mathrm{C}^{\prime}}=\omega_{\mathrm{A}}^{\mathrm{C}^{\prime}}-\omega_{\mathrm{B}}^{\mathrm{C}^{\prime}}$ and $\Delta \omega_{\mathrm{AB}}^{\mathrm{N}}=\omega_{\mathrm{A}}^{\mathrm{N}}-\omega_{\mathrm{B}}^{\mathrm{N}}$ are the changes in isotropic chemical shifts experienced by the $\mathrm{C}^{\prime}$ and $\mathrm{N}$ nuclei when jumping between conformations $\mathrm{A}$ and B. Likewise, there may be two different conformations $\mathrm{A}^{\prime}$ and $\mathrm{B}^{\prime}$ in the HOLO form, with $\tau_{\mathrm{ex}}^{\prime}=1 /\left(k_{\mathrm{A}^{\prime} \mathrm{B}^{\prime}}+k_{\mathrm{B}^{\prime} \mathrm{A}^{\prime}}\right)$, leading to a HOLO rate $R_{i}^{\prime C S M} / \mathrm{CSM}$ defined in analogy to Equation (4). The rates $R_{i}{ }^{\mathrm{CSM} / \mathrm{CSM}}$ (APO) and ${R_{i}^{\prime}}^{\mathrm{CSM} / \mathrm{CSM}}$ (HOLO) may be either positive or negative depending on whether the isotropic shifts of the $\mathrm{C}^{\prime}$ and $\mathrm{N}$ nuclei are correlated or anti-correlated, i.e., whether local motions cause chemical shifts to move in the same or in opposite directions upon a jump between conformations $\mathrm{A}$ and $\mathrm{B}$, or between $\mathrm{A}^{\prime}$ and $\mathrm{B}^{\prime}$. 
Equation (4) is analogous to the behaviour of exchange contributions to the line-widths of singlequantum spectra. In fact, the CSM/CSM rate is analogous to an $R_{\mathrm{ex}} / R_{\mathrm{ex}}$ cross-correlation rate.

In this paper, we shall focus attention on changes in cross-correlation rates upon complex formation:

$$
\begin{aligned}
\Delta R_{i}^{\mathrm{CSM} / \mathrm{CSM}}= & R_{i}^{\prime \mathrm{CSM} / \mathrm{CSM}}(\mathrm{HOLO}) \\
& -R_{i}^{\mathrm{CSM} / \mathrm{CSM}}(\mathrm{APO})
\end{aligned}
$$

which amounts to:

$$
\begin{aligned}
\Delta R_{i}^{\mathrm{CSM} / \mathrm{CSM}}= & 2 p_{\mathrm{A}^{\prime}} p_{\mathrm{B}^{\prime}} \tau_{\mathrm{ex}}^{\prime} \Delta \omega_{\mathrm{A}^{\prime} \mathrm{B}^{\prime}}^{\mathrm{C}^{\prime}} \Delta \omega_{\mathrm{A}^{\prime} \mathrm{B}^{\prime}}^{\mathrm{N}}(\mathrm{HOLO}) \\
& -2 p_{\mathrm{A}} p_{\mathrm{B}} \tau_{\mathrm{ex}} \Delta \omega_{\mathrm{AB}}^{\mathrm{C}^{\prime}} \Delta \omega_{\mathrm{AB}}^{\mathrm{N}}(\mathrm{APO})
\end{aligned}
$$

Clearly, to the extent that Equation (4) is applicable, changes $\Delta R_{i}^{\mathrm{CSM} / \mathrm{CSM}}$ can arise either from changes in 'dynamic' parameters (populations $p_{\mathrm{A}}$, $p_{\mathrm{B}}$ and residence time $\tau_{\mathrm{ex}}$ ) or from changes in 'static' parameters $\Delta \omega_{\mathrm{AB}}^{\mathrm{C}^{\prime}}$ and $\Delta \omega_{\mathrm{AB}}^{\mathrm{N}}$ that arise either from structural differences between the conformations $\mathrm{A}$ and $\mathrm{B}$ or $\mathrm{A}^{\prime}$ and $\mathrm{B}^{\prime}$ in the $\mathrm{APO}$ or HOLO forms, or from chemical shift changes induced by ligand binding. The latter need not necessarily involve changes in structure between the APO and HOLO forms.

If it is assumed that the 'static' parameters $\Delta \omega_{\mathrm{AB}}^{\mathrm{C}^{\prime}}$ and $\Delta \omega_{\mathrm{AB}}^{\mathrm{N}}$ are independent of temperature (Palmer et al., 2001), while the dynamic parameters $p_{\mathrm{A}}, p_{\mathrm{B}}$ and $\tau_{\mathrm{ex}}$ obviously depend on the temperature, the temperature dependence of the $R_{i}^{\mathrm{CSM} / \mathrm{CSM}}$ rates allows one to separate dynamic and static factors. The temperature dependence of the $R_{i}^{\mathrm{CSM} / \mathrm{CSM}}$ rates can be determined from the measurement of experimental rates $R_{i}^{\exp }(T)$ as a function of temperature, followed by the subtraction of $R_{i}^{\mathrm{CSA} / \mathrm{CSA}}\left(T_{\mathrm{o}}\right)$ and $R_{i}^{\mathrm{DD} / \mathrm{DD}}\left(T_{\mathrm{o}}\right)$ at each temperature. Ideally, one should measure the temperature dependence of the isotropic shifts of both $\mathrm{C}^{\prime}$ and $\mathrm{N}$ nuclei in order to determine the CSA parameters as a function of $T$, and one should determine the (slight) variations of structure as a function of temperature. In practice however, we have found that it is sufficient to scale the $R_{i}^{\mathrm{CSA} / \mathrm{CSA}}\left(T_{\mathrm{o}}\right)$ and $R_{i}^{\mathrm{DD} / \mathrm{DD}}\left(T_{\mathrm{o}}\right)$ rates estimated at $T_{0}=308 \mathrm{~K}$ according to the ratio of the correlation times $\tau_{\mathrm{c}}(T) / \tau_{\mathrm{c}}\left(T_{0}\right)$. The temperature dependence of the correlation time can be derived from the Stokes-Einstein relationship:

$$
\tau_{\mathrm{c}}=\frac{4 \pi \eta_{\mathrm{w}} r_{\mathrm{H}}^{3}}{3 k_{\mathrm{B}} T}
$$

According to Atkins (1994), the viscosity of water $\eta_{\mathrm{w}}$ as a function of the temperature $t\left[{ }^{\circ} \mathrm{C}\right]$ can be expressed by $\eta_{\mathrm{w}}(t)=\exp (\mathrm{B} / \mathrm{A}) \cdot \eta_{\mathrm{w}}(20)$ with $\mathrm{A}=1.37023(t-20)+8.36 \cdot 10^{-4}(t-20)^{2}$ and $\mathrm{B}=109+t$. If the hydrodynamic radius $r_{\mathrm{H}}$ is assumed to be independent of temperature, the correlation time can be considered to be a linear function of $T$ over the range of interest, i.e.,

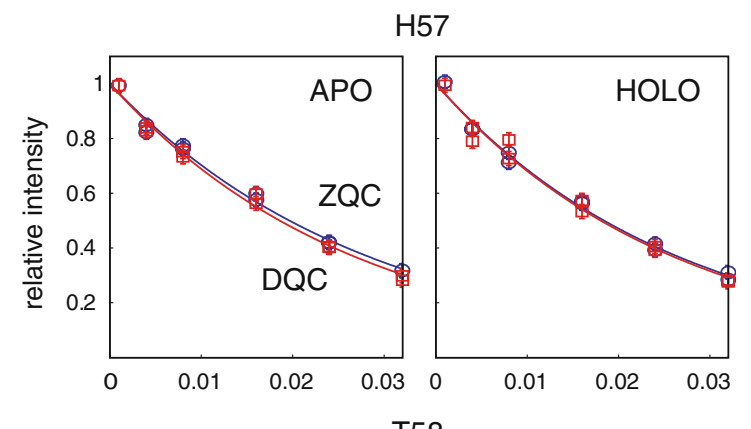

T58

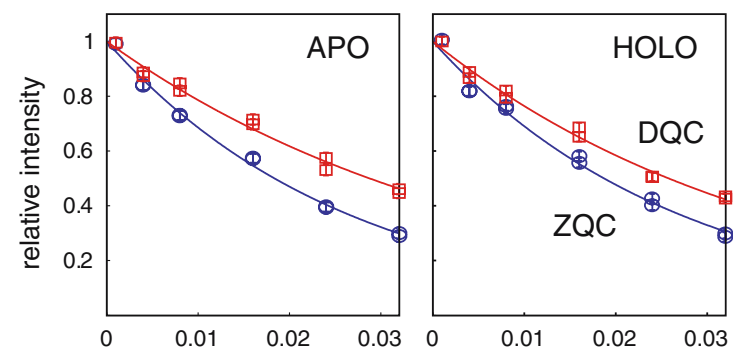

V59

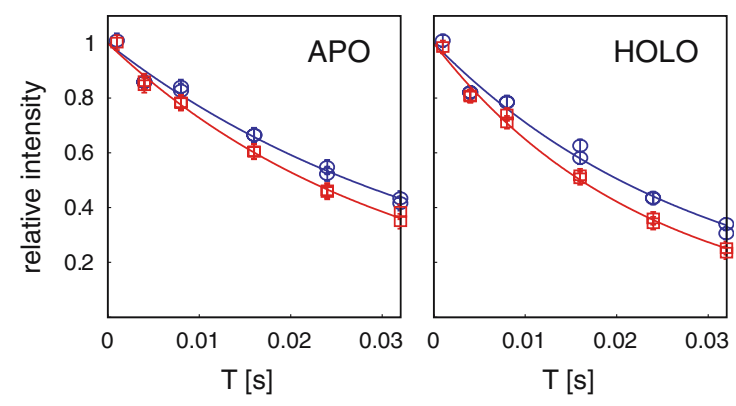

Figure 1. Three experimental decays of $\mathrm{DQC}\left(\mathrm{C}_{+}^{\prime} \mathrm{N}_{+}\right)$(red squares) and $\mathrm{ZQC}\left(\mathrm{C}_{+}^{\prime} \mathrm{N}_{-}\right)$(blue circles) for His57, Thr58 and Val59, in APO-MUP (left) and HOLO-MUP (right), i.e., with the pheromone IBMP as ligand. 

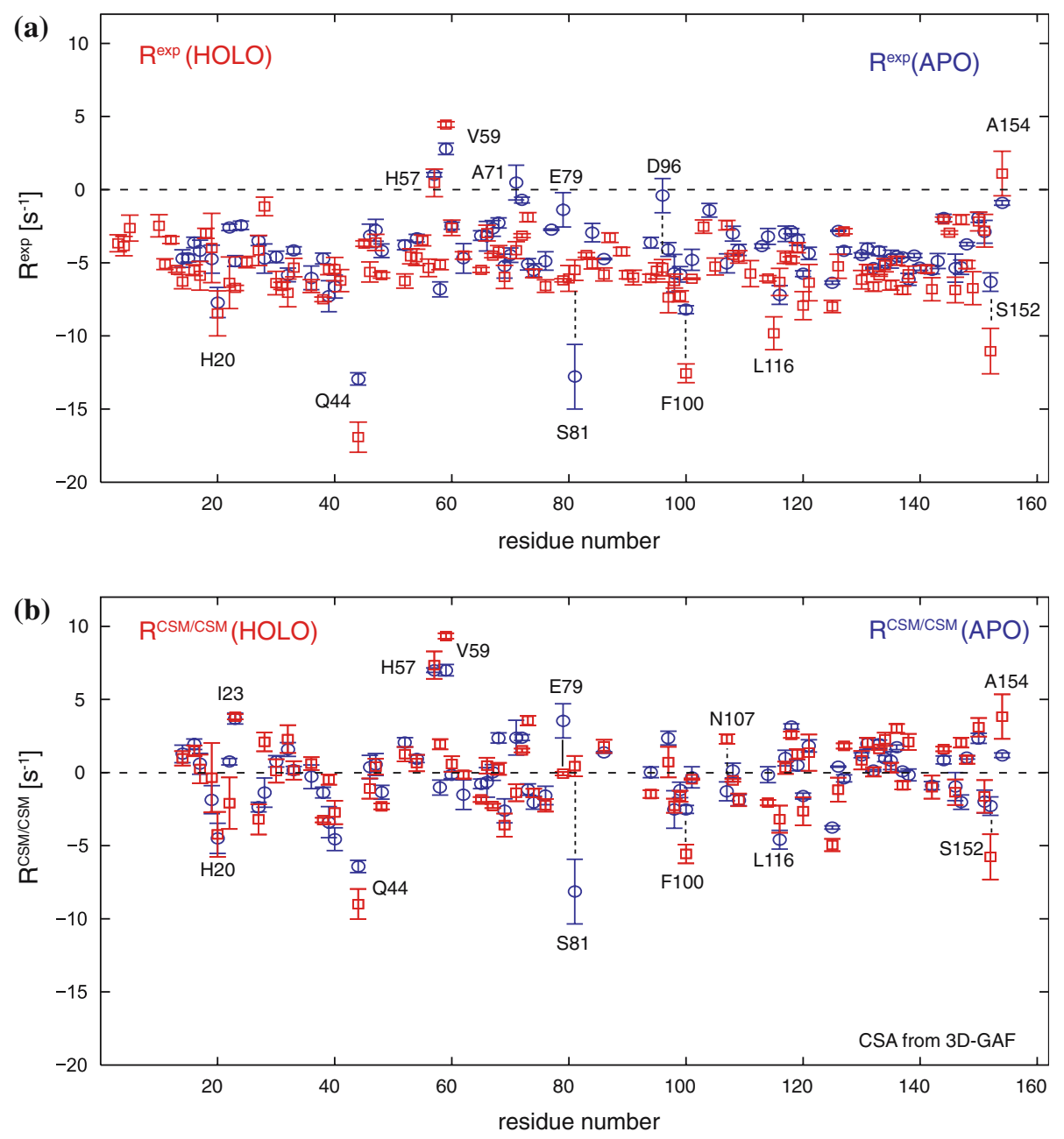

Figure 2. (a) 'Raw' experimental rates $R_{i}^{\exp }$ as defined in Equation (1) for APO-MUP (blue circles) and HOLO-MUP (red squares) measured at $T=308 \mathrm{~K}$ and $600 \mathrm{MHz}$; (b) Contributions $R_{i}^{\mathrm{CSM} / \mathrm{CSM}}$ due to correlated isotropic chemical shift modulations obtained by subtracting estimated $R_{i}^{\mathrm{DD} / \mathrm{DD}}$ and $R_{i}^{\mathrm{CSA} / \mathrm{CSA}}$ rates from experimental relaxation rates according to Equation (3). The $R_{i}^{\mathrm{CSM} / \mathrm{CSM}}$ rates are only shown for APO-MUP (blue circles) and for HOLO-MUP (red squares) for all amino-acids that can be resolved in both HSQC spectra.

$\tau_{\mathrm{c}}^{\mathrm{HOLO}}(T)=-0.12 T+45.19 \mathrm{~ns} \quad$ and $\quad \tau_{\mathrm{c}}^{\mathrm{APO}}(T)$ $=-0.09 T+36.08 \mathrm{~ns}$.

\section{Results and discussion}

Figure 1 shows some typical experimental decays of double- (red squares) and zero-quantum coherences (blue circles) recorded at $308 \mathrm{~K}$.

Figure 2a shows the 'raw' experimental relaxation rates $R_{i}^{\exp }$ for all accessible residues in APO-MUP (blue circles) and HOLO-MUP (red squares). The $R_{i}^{\mathrm{DD} / \mathrm{DD}}$ and $R_{i}^{\mathrm{CSA} / \mathrm{CSA}}$ rates, esti- mated at $308 \mathrm{~K}$ as discussed above, and given in the Supplementary Material, are shown schematically as histograms in Figure S3. Of a total of 117 observable residues in the APO form, 16 had to be discarded because of overlapping signals, and 12 because of poor signal-to-noise, so that 89 residues could be retained. The 'raw' experimental rates in the APO form range between -12.9 and $+2.8 \mathrm{~s}^{-1}$. Applying the same criteria for the HOLO form, 23 of the 133 observable residues had to be excluded because of overlapping signals, and 2 because of poor signal-to-noise, so that 108 rates could be analysed. The 'raw' experimental rates of the 
HOLO form lie in a range between -16.9 to $+4.5 \mathrm{~s}^{-1}$. Figure $2 \mathrm{~b}$ shows 'corrected' relaxation rates $R^{\mathrm{cs} / \mathrm{cs}} T=R_{i}^{\mathrm{CSM} / \mathrm{CSM}}$ at $308 \mathrm{~K}$ after subtraction of the $R_{i}^{\mathrm{DD} / \mathrm{DD}}$ and $R_{i}^{\mathrm{CSA} / \mathrm{CSA}}$ contributions according to Equation (3) for APO-MUP (blue circles) and HOLO-MUP (red squares).

As discussed above, it is not possible to derive dynamic information directly from the difference $\Delta R_{i}^{\mathrm{CSM} / \mathrm{CSM}}$ between the APO and HOLO forms, since these comprise an admixture of 'dynamic' parameters (populations $p_{\mathrm{A}}, p_{\mathrm{B}}$ and residence time $\tau_{\mathrm{ex}}$ ) and 'static' parameters $\Delta \omega_{\mathrm{AB}}^{\mathrm{C}^{\prime}}$ and $\Delta \omega_{\mathrm{AB}}^{\mathrm{N}}$. However, if it is assumed that the static parameters are independent of temperature (Palmer et al., 2001), the derivative $\mathrm{d} R_{i}^{\mathrm{CSM} / \mathrm{CSM}}(T) / \mathrm{d} T$ with respect to temperature provides information about changes in dynamics between the APO and HOLO forms.

Figure $3 \mathrm{a}$ shows the temperature dependence of the experimental rates $R^{\mathrm{CS} / \mathrm{CS}}(T)=R_{i}^{\exp }(T)$ of Lys14 in APO- and HOLO-MUP. In this example, the subtraction of the estimated dipolar and CSA contributions according to Equation (3) leads to the conclusion that the corrected rates $R_{i}^{\mathrm{CSM} / \mathrm{CSM}}(T)$ in Figure $3 \mathrm{~b}$ vanish for both APOand HOLO-MUP over the full temperature range that could be investigated. This means that for this residue there are no significant dynamic effects on the time-scale of interest. Other residues have rates whose absolute value decreases with increasing temperature, in analogy to the $R_{\mathrm{ex}}$ contribution to transverse ${ }^{15} \mathrm{~N}$ relaxation in the fast exchange regime. In His57 (Figure 3c), the temperature dependence of both APO- and HOLO-MUP forms are very pronounced, but complexation has no visible effect. In Thr58 (Figure 3d), the temperature dependence is less pronounced and the slopes are conserved, but the intercepts are clearly affected by complexation. In Phe100 (Figure 3e), both slopes and intercepts are modified upon complexation with the pheromone. Finally, Gln44 (Figure 4f) shows a linear temperature dependence in APO-MUP and a highly non-linear behaviour in the HOLO form, indicating that some dynamic process is dramatically affected by complexation at $295.6 \mathrm{~K}$. In principle, if the logarithms of the rates were plotted as a function of $1 / T$, the slopes would correspond to activation energies, but the quality of the data does not allow one to draw quantitative conclusions at this stage.
It is instructive to examine the rates $R_{i}^{\mathrm{CSM} / \mathrm{CSM}}$ for APO and HOLO MUP (Figure 2b): His57 resides within the ' $\mathrm{C}-\mathrm{D}$ ' loop, while Val59 is adjacent to it. This loop has been proposed to be the site of interaction with a cell-surface receptor in the structurally related retinol-binding protein (Sivaprasadarao and Findlay, 1994). Interestingly, $\mathrm{d} R_{i}^{\mathrm{CSM} / \mathrm{CSM}}(T) / \mathrm{d} T$ remains large for these residues in both forms of the protein (Figure 4a), suggesting that the rates can be attributed to slow time-scale dynamics.

Moreover, the dynamics of Val 59 differ markedly between the APO and HOLO forms (Figure 4b), which is consistent with an earlier observation based on conventional ${ }^{15} \mathrm{~N}$ relaxation measurements (Bingham et al., 2004) that residues in this region of the protein 'melt' on binding with the IBMP ligand. Thus the dynamics of this region of the protein are perturbed over a wide range of time scales on ligand binding.

A second loop in the region of Phe100 exhibits substantially enhanced relaxation only in the HOLO form of the protein (Figure 2), and shows an enhanced derivative $\mathrm{d} R_{i}^{\mathrm{CSM} / \mathrm{CSM}}(T) / \mathrm{d} T$ upon complexation (Figure 4b). This region of the protein is known from ${ }^{15} \mathrm{~N}$ relaxation measurements (Bingham et al., 2004) to 'freeze' on ligand binding. In contrast, other dynamic differences between APO and HOLO forms implied by the data in Figure 4, e.g., regions surrounding Arg39, Gln44, Gly121 and Leu126 are not observed in ${ }^{15} \mathrm{~N}$ relaxation measurements, suggesting that slow time-scale dynamic processes are dominant in these cases. The enhancement of the derivative $\mathrm{d} R_{i}^{\mathrm{CSM} / \mathrm{CSM}}(T) / \mathrm{d} T$ for Gln 44 is surprising in view of the fact that this residue is buried within a $\beta$-sheet region of the protein (Figure 5).

\section{Conclusions}

Upon binding to 2-methoxy-3-isobutylpyrazine (IBMP), major urinary protein (MUP) undergoes significant changes in slow internal dynamics. Our observations, based on relaxation rates of $\mathrm{C}^{\prime} \mathrm{N}$ double- and zero-quantum coherences, provide evidence of significant changes in correlated internal dynamics in the protein upon ligand binding, on time scales that have not been explored so far. 

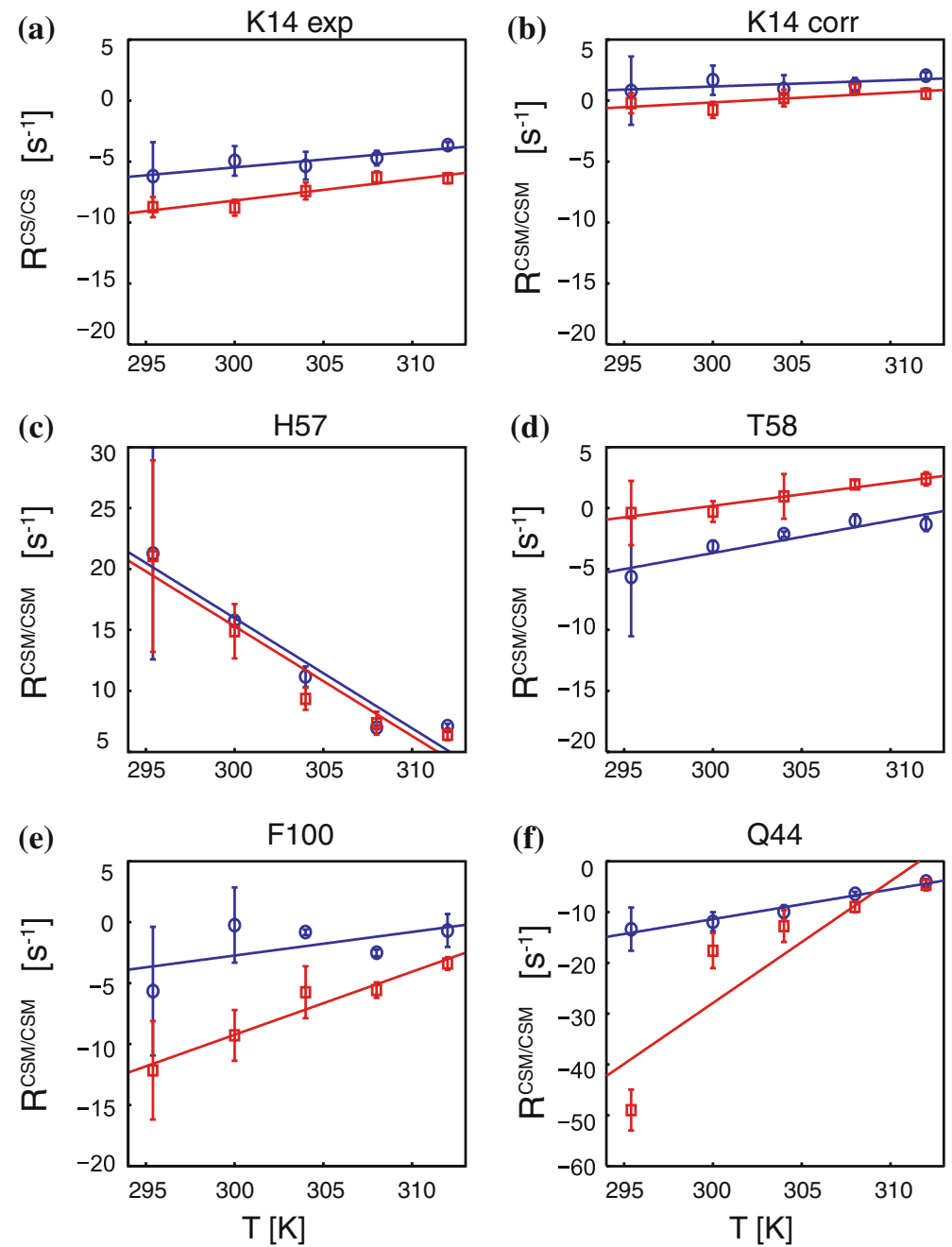

Figure 3. (a) Experimental rates, $R^{\mathrm{CS} / \mathrm{CS}}(T)=R_{i}^{\text {exp }}(T)$ for Lys14 in APO-MUP (blue circles) and HOLO-MUP (red squares) measured at $600 \mathrm{MHz}$ and $T=294.5,300,304,308$ and $312 \mathrm{~K}$. (b) Corrected rates $R_{i}^{\mathrm{CSM} / \mathrm{CSM}}(T)$ for Lys14 obtained after subtracting estimated DD/DD and CSA/CSA rates with a temperature dependence given by Equation (7). In this example, $R_{i}^{\mathrm{CSM} / \mathrm{CSM}} \approx 0$ at all temperatures, indicating the absence of any internal dynamics which is typical for many residues. (c)-(f) corrected rates $R_{i}^{\mathrm{CSM} / \mathrm{CSM}}(T)$ for His57, Thr58, Phe100, and Gln44, obtained by subtracting estimated temperature-dependent DD/DD and CSA/CSA rates. For His57 and Thr58, the slopes are not affected by complexation, while Gln44 and, to a lesser extent, Phe100 feature internal dynamics that are strongly affected by the presence of the pheromone. Note that all rates $R_{i}^{\operatorname{CSM} / \mathrm{CSM}}(T)$ tend to vanish at high temperatures (fast exchange); the signs are negative if the chemical shift modulations $\Delta \omega_{\mathrm{C}^{\prime}}$ and $\Delta \omega_{\mathrm{N}}$ are anti-correlated.

As with conventional backbone ${ }^{15} \mathrm{~N}$ relaxation measurements on MUP (Bingham et al., 2004), there are no obvious correlations between changes in rates $R_{i}^{\mathrm{CSM} / \mathrm{CSM}}$ and the location of the residues with respect to the binding site. However, these slow time-scale dynamic changes will clearly contribute to the entropy of ligand binding, and quantitation of the magnitude of these contributions is currently under investigation.

\section{Supporting information available}

Decay rates $R_{i}^{\exp }(\mathrm{APO})$ and $R_{i}^{\exp }(\mathrm{HOLO})$ measured at $T=308 \mathrm{~K}$ and $\mathrm{B}_{0}=14 \mathrm{~T}(600 \mathrm{MHz})$, calculated contributions to the dipolar relaxation rates $R_{i}^{\mathrm{DD} / \mathrm{DD}}$, and $R_{i}^{\mathrm{CSA} / \mathrm{CSA}}$ rates estimated from the isotropic shifts and the empirical rules derived by Loth et al. (2005) for five different motional models. Decays rates for APO- and HOLO-MUP 

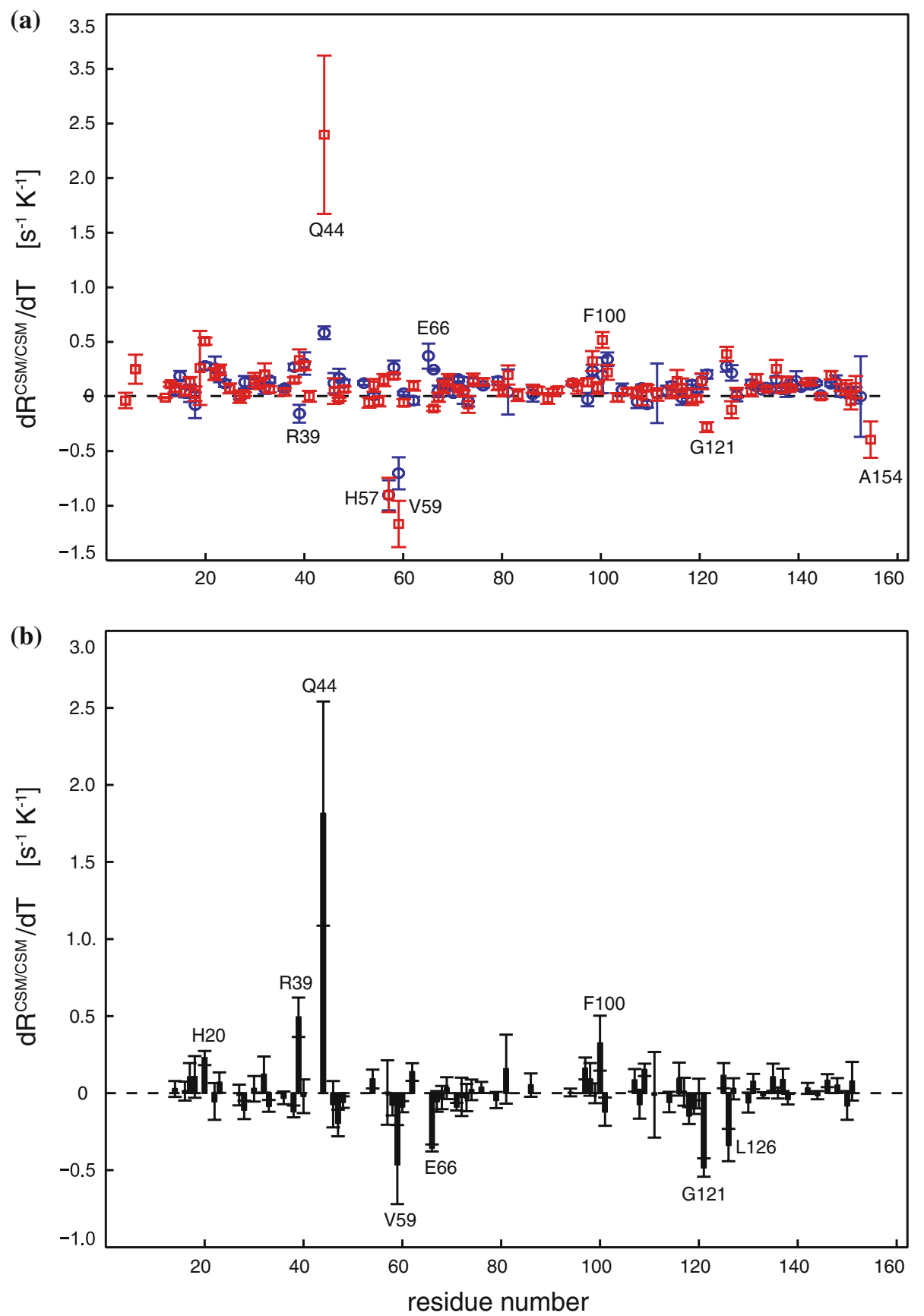

Figure 4. (a) Derivatives $\mathrm{d} R_{i}^{\mathrm{CSM} / \mathrm{CSM}}(T) / \mathrm{d} T$ for all amino-acids where the signals could be followed as a function of temperature for both APO-MUP (blue circles) and HOLO-MUP (red squares). (b) Differences in the derivatives between the HOLO and APO forms.

measured at five different temperatures: $T=205.4$, 300, 304, 308 and 312 K. Sequence used for the determination of auto-relaxation rates of doubleand zero-quantum coherences involving the amide nitrogen $\mathrm{N}$ of the $i$ th amino acid and the carbonyl carbon $\mathrm{C}^{\prime}$ of the $(i-1)$ th residue. Partial ${ }^{15} \mathrm{~N},{ }^{1} \mathrm{H}$ correlation spectra ('HSQC') with some assignments of APO- and of HOLO-MUP. Histogram 


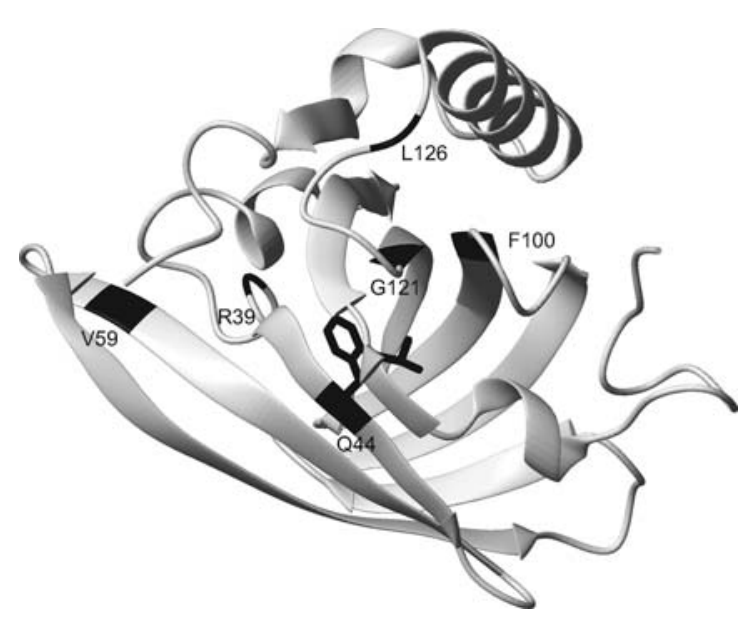

Figure 5. Ribbon representation of the X-ray structure of HOLO-MUP, where the aromatic ring of the pheromone 2methoxy-3-isobutylpyrazine (IBMP) appears in the centre. Residues whose derivatives $\mathrm{d} R_{i}^{\mathrm{CSM} / \mathrm{CSM}}(T) / \mathrm{d} T$ are enhanced upon ligand binding are emphasized in black. The figure was obtained with the program MOLMOL (Koradi et al., 1996).

and tables showing estimated DD/DD and CSA/ CSA rates. This electronic supplementary material is available at http://dx.doi.org/10.1007/s10858005-3355-y.

\section{Acknowledgements}

Stimulating discussions with Philippe Pelupessy and Jens Dittmer are acknowledged. This work has been supported by the European Union (Research Training Network on Cross-Correlations), the Fonds National de la Recherche Scientifique (FNRS, Switzerland), the Commission pour la Technologie et l'Innovation (CTI, Switzerland), the Centre National de la Recherche Scientifique (CNRS, France), and the BBSRC (grant no. B19388 to SWH, United Kingdom).

\section{References}

Arnold, W.D. and Oldfield, E. (2000) J. Am. Chem. Soc., , 122, 12835-12841.
Atkins, P.W. (1994) Physical Chemistry, Oxford University Press.

Berman, H.M., Westbrook, J., Feng, Z., Gilliland, G., Bhat, T.N., Weissig, H., Shindyalov, I.N. and Bourne, P.E. (2000) Nucleic Acids Res., 28, 235-242.

Bingham, R., Bodenhausen, G., Findlay, J.H.B.C., Hsieh, S.Y., Kalverda, A.P., Kjellberg, A., Perazzolo, C., Phillips, S.E.V., Seshadri, K., Turnbull, W.B. and Homans, S.W. (2004) J. Am. Chem. Soc., 126, 1675-1681.

Bytchenkoff, D., Pelupessy, P. and Bodenhausen, G. (2005) J. Am. Chem. Soc., 127, 5180-5185.

Delaglio, F., Grzesiek, S., Vuister, G.W., Zhu, G., Pfeifer, J. and Bax, A. (1995) J. Biomol. NMR, 6, 277-293.

Dittmer, J. and Bodenhausen, G. (2004) J. Am. Chem. Soc., 126, 1314-1315.

Frueh, D. (2002) Prog. NMR Spectrosc., 41, 305-324.

Frueh, D., Tolman, J.R., Bodenhausen, G. and Zwahlen, C. (2001) J. Am. Chem. Soc., 123, 4810-4816.

Grzesiek, S. and Bax, A. (1992) J. Am. Chem. Soc., 114, 62916293.

Kay, L.E., Ikura, M., Tschudin, R. and Bax, A. (1990) J. Magn. Reson., 89, 496-514.

Kay, L.E., Muhandiram, D.R., Wolf, G., Shoelson, S.E. and Forman-Kay, J.D. (1998) Nat. Struct. Biol., 5, 156-163.

Koradi, R., Billeter, M. and Wuthrich, K. (1996) J. Mol. Graph., 14, 51 .

Krízová, H., Zídek, L., Stone, M.J., Novotny, M.V. and Sklenar, V. (2004) J. Biomol. NMR, 28, 369-384.

Lee, A.L., Kinnear, S.A. and Wand, A.J. (2000) Nat. Struct. Biol., 7, 72-77.

Lipari, G. and Szabo, A. (1982a) J. Am. Chem. Soc., 104, 45594570.

Lipari, G. and Szabo, A. (1982b) J. Am. Chem. Soc., 104, 45464559.

Loth, K., Pelupessy, P. and Bodenhausen, G. (2005) J. Am. Chem. Soc., 127, 6062-6068.

Muhandiram, D.R. and Kay, L.E. (1994) J. Magn. Reson., B103, 203-216.

Palmer, A.G., Kroenke, C.D. and Loria, J.P. (2001) Methods Enzymol., 339, 204-238.

Pellecchia, M., Pang, Y.X., Wang, L.C., Kurochkin, A.V.Kumar, A. and Zuiderweg, E.R.P. (1999) J. Am. Chem. Soc., 121, 9165-9170.

Sivaprasadarao, A. and Findlay, J.B.C. (1994) Biochem. J., 300, 437-442.

Wist, J., Frueh, D., Tolman, J.R. and Bodenhausen, G. (2004) J. Biomol. NMR, 28, 263-272.

Wist, J., Perazzolo, C. and Bodenhausen, G. (2005) Appl. Magn. Res., 29, 251-259.

Zídek, L., Novotny, M.V. and Stone, M.J. (1999) Nat. Struct. Biol., 6, 1118-1121. 